\title{
SOME RESULTS IN THE THEORY OF $H$-SPACES
}

BY J. R. HUBBUCK

Communicated by P. E. Thomas, May 13, 1968

1. We study $H$-spaces which are connected finite complexes, with $H_{*}(X, Z)$ free of 2 -torsion, by considering the complex $K$-theory of their projective planes. In particular we show that no 7 sphere-bundle over an 11 or 15 sphere can be an $H$-space, ${ }^{1}$ and that a homotopy associative, homotopy commutative $H$-space must have the homotopy type of a torus.

2. Let $X$ be an $H$-space as above, then $H^{*}(X, Q)$ is an exterior algebra on odd dimensional generators, and we assume that the Hopf algebra structure, with comultiplication induced by the $H$-space map, is primitively generated. The number of generators we call the rank of $X$ and the dimensions in which they occur, the type of $X$.

THEOREM 1. Let $H^{*}(X, Q)$ have not more than one generator in any one dimension, then

(i) If the rank of $X$ is 1, its type is 1,3 or 7 .

(ii) If the rank of $X$ is 2 , its type is $(1,3)(1,7)(3,5)$ or $(3,7)$.

(iii) If the type of $X$ contains $4 m-1$, with $m$ not divisible by 4 , then its type contains $4 q-1$, for all $q$ with $2 \leqq q \leqq m$.

(iv) If the type of $X$ contains $2 m-1$, with $m$ odd, then it contains $2 q-1$, for all $q$ with $2 \leqq q \leqq m$.

Part (i) is well known. It was first proved in [3] and we have generalized the proof of [5]. Part (ii) answers questions raised in [4] and [8]. It was the solution of this problem that led to the rest of this work. In general, if you specify the number of generators, better results can be obtained by applying the methods of this paper rather than the results.

3. Let $X$ be a noncontractible connected finite complex which is a homotopy commutative $H$-space, then $H_{*}(X, Z)$ is free of 2-torsion by Theorem 8.5 of [7].

THEOREM 2. Let $X$ be a homotopy associative, homotopy commutative $H$-space, then $X$ has the homotopy type of a torus.

${ }^{1}$ Added in pronf. Dr. R. Douglas and Dr. F. Sigrist have recently announced their independent proof of this result. 
This is not the strongest result which we can prove. We may eliminate the condition of homotopy associativity, using recent work of James, but for technical reasons we have to use methods other than those outlined here.

4. We develop a simple axiomatic theory for dealing with these and similar questions, motivated by complex $K$-theory. We sketch this briefly in the case of the prime 2 , and write $Q_{2}$ for the subring of rationals with odd denominators.

Let $M$ be a finitely generated $Q_{2}$-module which is also a commutative filtered ring, whose associated graded ring is torsion free. We suppose that we have filtration preserving ring homomorphisms $\Psi^{k}: M \rightarrow M$, for each $k$, with $\Psi^{k} \Psi^{m}=\Psi^{m} \Psi^{k}$ and $\Psi^{2}(x)=x^{2} \bmod 2$, which correspond to the Adams operators of [2]. We also assume an integrality condition, motivated by the Integrality Theorem on the Chern character of [1].

Since $N$, the associated graded ring is torsion free, $N$ and $M$ are isomorphic as filtered $Q_{2}$-modules, and we take a particular choice for this isomorphism, $J: N \rightarrow M$ say. Using the $\Psi^{k}$, we define homomorphisms $S_{J}^{r}: N_{n} \rightarrow N_{n+r}$, which behave something like the Steenrod squares in cohomology. They satisfy certain Adem-type relations, but not just $\bmod 2$, in general $\bmod 2^{n}$ for all $n$. We also have generalized Cartan formulae, in particular if $J$ is a ring isomorphism, we have a $Q_{2}$ Cartan formula.

Let $N$ be a truncated polynomial algebra of height 3 on generators no two of which have the same dimension. Our main result is,

Theorem 3.

(i) If $N$ has one generator, then it has dimension 1, 2 or 4.

(ii) If $N$ has two generators, then they have dimensions $(1,2)(1,4)$, $(2,3)$, or $(2,4)$.

(iii) If $N$ has a generator of dimension $2 m$, with $m$ not divisible by 4 , then it has generators of dimension $2 q$ for all $q$ with $2 \leqq q \leqq m$.

(iv) If $N$ has a generator of dimension $2 m-1$, then it has generators of all dimensions less than $2 m-1$ and greater than 1 .

In a large number of cases (iii) can be strengthened to show that there are always generators of dimension $2 q$ for $1 \leqq q \leqq m$, but we have not been able to prove this in general.

5. Our application is to take $M=K(X)$, complex $K$-theory with $Q_{2}$-coefficients and the CW-filtration. $N$ is then $H^{\text {even }}\left(X, Q_{2}\right)$, since $H_{*}(X, Z)$ is free of 2 -torsion.

In particular with the conditions of $\S 2$, if we take $M=K\left(P_{2} X\right)$, 
where $P_{2} X$ is the projective plane of $X$, Theorem 1 follows from Theorem 3.

The proof of Theorem 2 is quite simple using the results of $\$ 4$. We show that the $H$-squaring map is not an $H$-map unless all generators of $H^{*}(X, Q)$ have dimension 1 , once more using the projective plane of an $H$-space.

6. Acknowledgments. The contents of this note are some of the results prepared for a doctoral thesis at Oxford. I must thank Professor Adams for giving me considerable assistance in clarifying many of the technical details. In fact, when we consider corresponding results for odd primes, the best proofs now rely upon results proved by Professor Adams. I must also thank my supervisor, Dr. James, who first suggested that I should study $H$-spaces of rank 2 , and who has given constant encouragement and guidance.

\section{REFERENCES}

1. J. F. Adams, On Chern characters and the structure of the unitary group, Proc. Cambridge Philos. Soc. 57 (1961), 189-199.

2. - - Vector fields on spheres, Ann. of Math. 75 (1962), 603-632.

3. - On the nonexistence of elements of Hopf invariant one, Ann. of Math. 72 (1960), 20-104.

4. - - H-spaces with few cells, Topology 1 (1962), 67-72.

5. J. F. Adams and M. F. Atiyah, K-theory and the Hopf invariant, Quart. J. Math. Oxford Ser. 17 (1966), 31-38.

6. M. F. Atiyah, Power operations in K-theory, Quart. J. Math. Oxford Ser. 17 (1966), 165-193.

7. W. Browder, Homotopy commutative H-spaces, Ann. of Math 75 (1962), 283311.

8. W. Browder and E. Thomas, On the projective plane of an $H$-space, Illinois J. Math. 7 (1963), 492-502.

9. I. M. James, On sphere-bundles over spheres, Comment. Math. Helv. 35 (1961), 126-135.

MANChESTER UNIVERSITY 